Research Paper

\title{
Over-Expression of Alpha-Enolase as a Prognostic Biomarker in Patients with Pancreatic Cancer
}

\author{
Lichao Sun ${ }^{1 凶}$, Chunguang Guo ${ }^{3}$, Jianzhong $\mathrm{Cao}^{4}$, Joseph Burnett ${ }^{2}$, Zhihua Yang ${ }^{1}$, Yuliang Ran ${ }^{1}$, Duxin Sun ${ }^{2}, 5 \bowtie$ \\ 1. State Key Laboratory of Molecular Oncology, National Cancer Center/Cancer Hospital, Chinese Academy of Medical Sciences, Peking Union Medical \\ College, Beijing, China; \\ 2. Department of Pharmaceutical Sciences, University of Michigan, Ann Arbor, MI 48109, USA; \\ 3. Department of abdominal surgical oncology, Cancer Institute (Hospital), Chinese Academy of Medical Sciences, Peking Union Medical College, Beijing \\ 100021, China; \\ 4. The department of radiotherapy, The affiliated cancer hospital of Shanxi medical university, Taiyuan, Shanxi, 030013, China; \\ 5. College of Pharmacy, Tianjin Medical University, Tianjin, 300070, China.
}

$\triangle$ Corresponding authors: Lichao Sun, PhD, State Key Laboratory of Molecular Oncology, Cancer Hospital, Chinese Academy of Medical Sciences, Peking Union Medical College, Beijing, 100021, P. R. China Prof_sunlichao@163.com Duxin Sun, PhD, Department of Pharmaceutical Sciences, University of Michigan, Ann Arbor, MI 48109. duxins@umich.edu

(c) Ivyspring International Publisher. This is an open access article distributed under the terms of the Creative Commons Attribution (CC BY-NC) license (https://creativecommons.org/licenses/by-nc/4.0/). See http://ivyspring.com/terms for full terms and conditions.

Received: 2016.12.12; Accepted: 2017.03.01; Published: 2017.06.22

\begin{abstract}
Background: Alpha-enolase is an important glycolytic enzyme, and its aberrant expression has been associated with multiple tumor progression. However, few studies investigated the expression of alpha-enolase and its clinical significance in pancreatic cancer (PC). Objectives: To evaluate alpha-enolase level in PC tissues by immunohistochemical (IHC) analysis, and investigate the association of alpha-enolase expression with clinicopathologic features. Methods: The alpha-enolase levels in pancreatic cancer tissues were analyzed by using the Oncomine database. The expression of alpha-enolase, Ki67 and p53 in pancreatic cancer and adjacent normal tissues were evaluated by $\mathrm{IHC}$ using the corresponding primary antibodies on the commercial tissue arrays. We also examined their association with clinicopathologic parameters, and explored their prognostic value in PC. Results: We identified an elevation of alpha-enolase mRNA level in pancreatic cancer independent datasets from Oncomine. IHC analysis showed that alpha-enolase protein levels were elevated in $47 \%(n=100)$ PC tissue samples, but there was weak or no staining in the normal tissues. Statistical analysis revealed that high levels of alpha-enolase were significantly associated with Stage and Lymph node metastasis. Correlation analysis indicated that over-expression of alpha-enolase was positively associated with Ki67 expression and inversely correlated with p53 expression. Furthermore, membranous expression of alpha-enolase was also observed in $29.8 \%$ (14/47) total alpha-enolase positive samples, and was significantly associated with Lymph node metastasis. Kaplan-Meier survival analysis demonstrated that high total alpha-enolase expression was significantly associated with unfavorable survival, while membranous alpha-enolase expression was significantly associated with better survival of PC patients. Multivariate Cox analysis demonstrated that total alpha-enolase expression was an independent prognostic factor for PC patients.

Conclusions: Our results suggested that alpha-enolase level was significantly elevated in pancreatic cancer tissues, which was closely associated with PC progression. It might be a candidate target for targeted pancreatic cancer treatments.
\end{abstract}

Key words: Alpha-enolase, Pancreatic Cancer, Marker, Prognosis.

\section{Introduction}

Pancreatic cancer (PC) is among the leading cause of deaths with an overall 5-year survival rate of about $6 \%$ [1]. Although Gemcitabine was widely used in the treatment of patients with pancreatic cancer, the response rate is low. Targeted therapy has been effective against the most common cancer, but the number of targeted drugs for pancreatic cancer is extremely limited [2]. Identifying targets is an important prerequisite for the development of cancer targeted drugs. Therefore, it is necessary to identify novel cancer targets.

Alpha-enolase is a key multifunctional enzyme 
involving in the glycolytic pathway, and it would determine the distinct function depending on its subcellular localization. It has been implicated in a great number of diseases including infection, inflammation and cancer. Besides its role in glycolysis, alpha-enolase was over-expressed in many different types of cancer, and played key roles in cancer progression [3-5]. Furthermore, targeting alpha-enolase monoclonal antibody could significantly suppress lung metastases in an experimental animal model of pancreatic cancer [6].

Despite the potential implication of alpha-enolase in cancer progression, no previous studies have examined its level and clinical significance in pancreatic cancer tissues.

In this study, we aimed at evaluating alpha-enolase level in PC tissues by IHC analysis, and investigating the association of alpha-enolase expression with clinicopathologic features.

\section{Results}

\section{Higher Alpha-enolase mRNA level identified in pancreatic cancer using the Oncomine database}

To roundly investigate alpha-enolase level in pancreatic cancer tissues, we analyzed the independent datasets from Oncomine. The results showed that alpha-enolase mRNA levels in pancreatic cancer tissues were significantly higher than normal tissue in two independent dataset (Figure 1).

\section{Higher expression of alpha-enolase protein detected in pancreatic cancer tissues}

The protein expression levels of alpha-enolase in PC and adjacent normal tissues were examined by IHC analysis. As showed in the Figure 2, the alpha-enolase expression was evaluated in $47 \%$
(47/100) PC samples, but found weak or no staining in normal pancreatic tissues. Moreover, membranous expression of alpha-enolase was also observed in $29.8 \%(14 / 47)$ alpha-enolase positive samples. Statistical analysis indicated that high levels of total alpha-enolase expression was significantly associated with Lymph node involvement $(\mathrm{P}=0.032)$ and Stage $(\mathrm{P}=0.035)$. There was no significant association with other clinicopathologic variables (Table 1). Importantly, we also found that the location of alpha-enolase expression was significantly associated with Lymph node involvement $(\mathrm{P}=0.016)$ (Table 2).

Table 1. Correlation between total alpha-enolase expression in pancreatic cancer tissues and clinicopathological parameters

\begin{tabular}{llll}
\hline & \multicolumn{2}{l}{ Alpha-enolase } & \\
\cline { 2 - 3 } & negative & positive & p-value \\
\hline Gender (Male: Female) & $31: 22$ & $31: 16$ & 0.443 \\
Age & $61.2 \pm 11.7$ & $62.2 \pm 11.0$ & 0.648 \\
$\begin{array}{l}\text { Depth of invasion } \\
\text { T1+ T2 }\end{array}$ & 44 & 35 & 0.295 \\
T3+ T4 & 9 & 12 & \\
Lymph node involvement & & & $0.032^{*}$ \\
N0 & 35 & 21 & \\
N1 & 18 & 26 & \\
Distant metastasis & & & 0.129 \\
M0 & 53 & 45 & \\
M1 & 0 & 2 & \\
Stage & & & $0.035^{*}$ \\
1 & 28 & 15 & \\
2+3+4 & 25 & 32 & \\
$\begin{array}{l}\text { Grade } \\
\text { 1+2 }\end{array}$ & & & 0.385 \\
3 & 49 & 41 & \\
Ki67 & 4 & 6 & \\
negative \\
positive
\end{tabular}

A

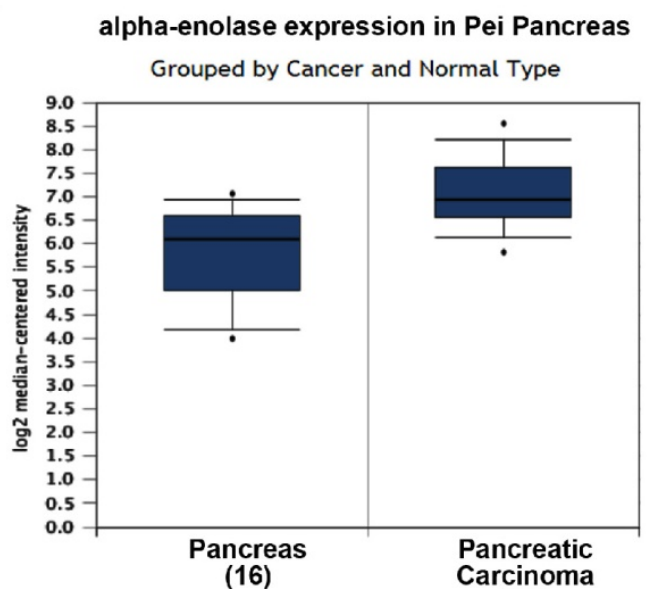

B

alpha-enolase expression in Logsdon Pancreas Grouped by Cancer and Normal Type

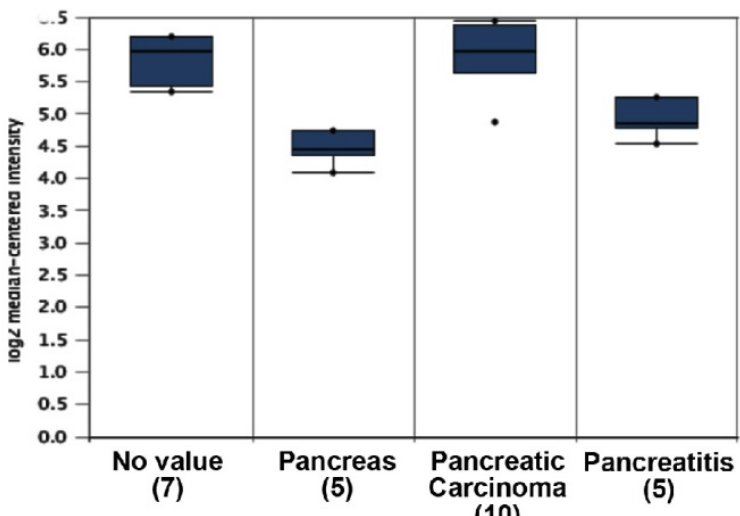

Figure 1. alpha-enolase mRNA level in human pancreatic cancers using the Oncomine database. A. alpha-enolase mRNA expression in Pei Pancreas dataset. B. alpha-enolase mRNA expression in Logsdon Pancreas dataset. 
A
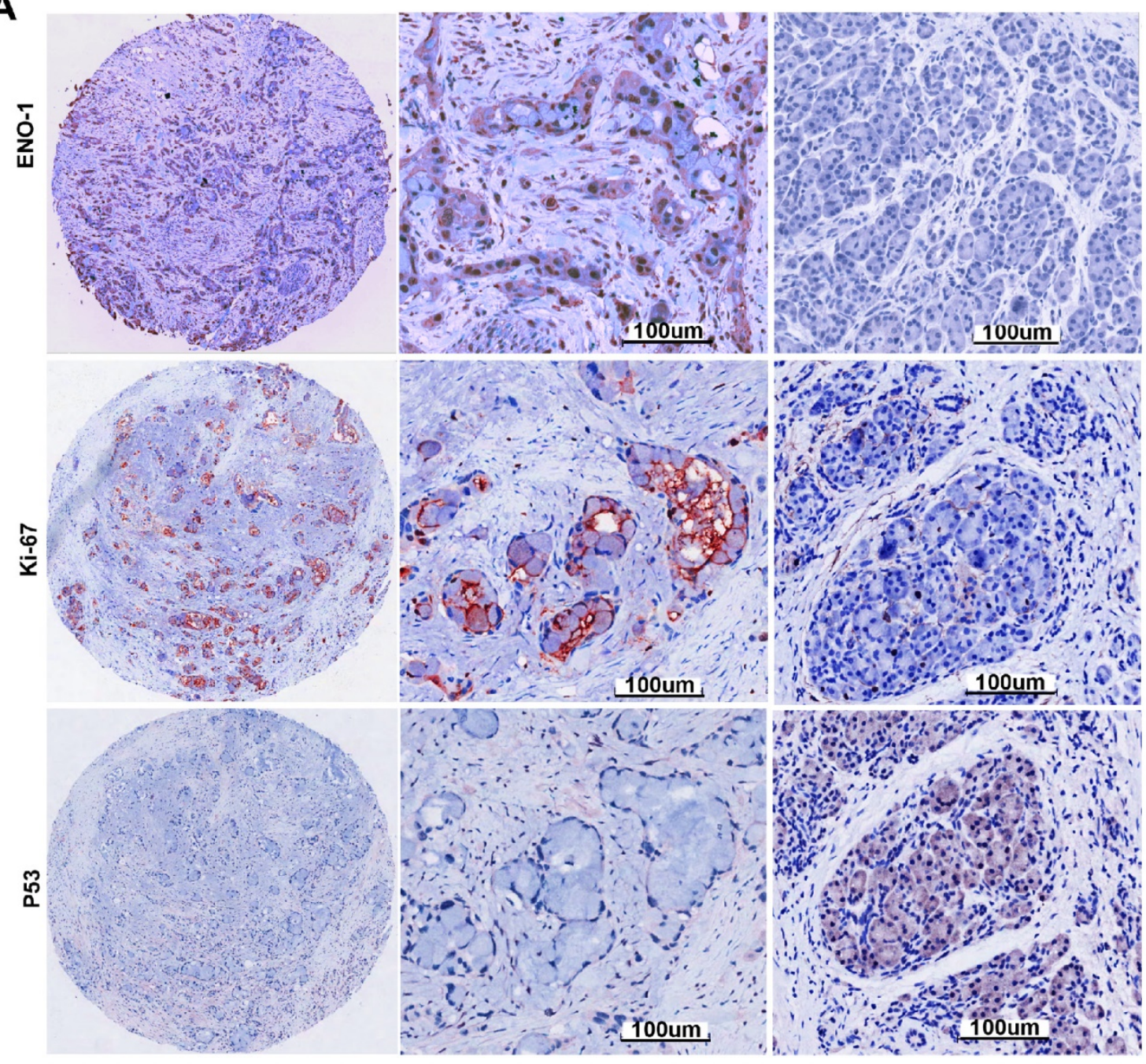

B

\section{Cancer}

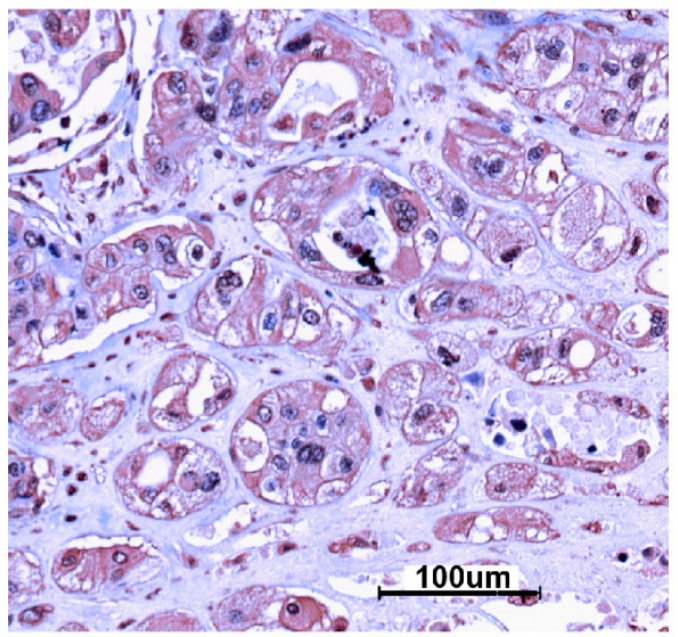

Alpha-enolase membrane staining

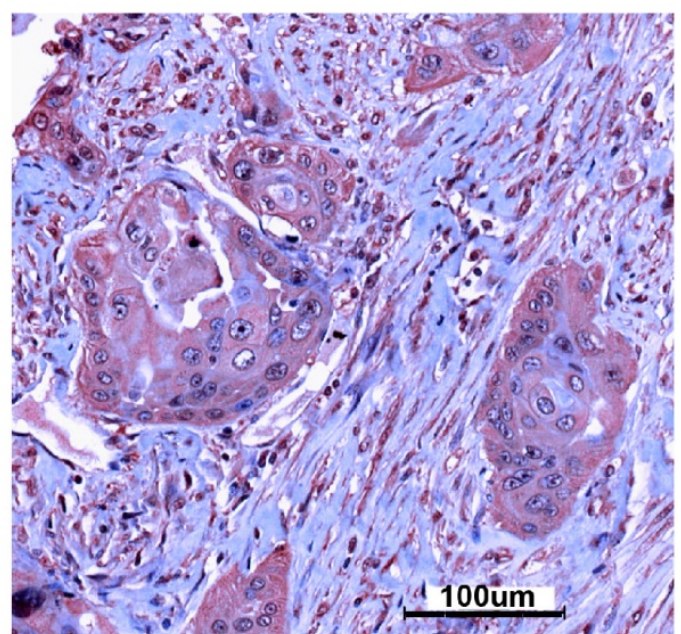

Alpha-enolase cytoplasmic staining

Figure 2. Alpha-enolase, p53 and ki67 expression in pancreatic cancer tissues were determined by immunochemistry. A. Positive expression of Alpha-enolase, p53 and ki67. B. alpha-enolase membranous and total expression of Alpha-enolase. 
Table 2. Correlation between alpha-enolase localization in pancreatic cancer tissues and clinicopathological parameters

\begin{tabular}{|c|c|c|c|}
\hline \multirow{3}{*}{ Depth of invasion } & \multicolumn{2}{|c|}{ Alpha-enolase } & \multirow[t]{2}{*}{ p-value } \\
\hline & Total & Membrane & \\
\hline & & & 0.297 \\
\hline & 26 & 9 & \\
\hline $\mathrm{T} 3+\mathrm{T} 4$ & 7 & 5 & \\
\hline Lymph node involvement & & & $0.016^{*}$ \\
\hline No & 11 & 10 & \\
\hline N1 & 22 & 4 & \\
\hline Distant metastasis & & & 0.523 \\
\hline M0 & 32 & 13 & \\
\hline M1 & 1 & 1 & \\
\hline Stage & & & 0.083 \\
\hline 1 & 8 & 7 & \\
\hline $2+3+4$ & 25 & 7 & \\
\hline Grade & & & 0.839 \\
\hline $1+2$ & 29 & 12 & \\
\hline 3 & 4 & 2 & \\
\hline Ki67 & & & 0.587 \\
\hline negative & 17 & 6 & \\
\hline positive & 16 & 8 & \\
\hline P53 & & & 0.414 \\
\hline negative & 22 & 11 & \\
\hline positive & 11 & 3 & \\
\hline
\end{tabular}

Table 3. The Correlation between alpha-enolase and Ki67, P53

\begin{tabular}{cll}
\hline & Ki67 & P53 \\
\hline alpha-enolase Correlation coefficient & $.223^{*}$ & $-0.343^{* *}$ \\
Sig. (2-tailed) & .020 & .000 \\
N & 100 & 100 \\
\hline
\end{tabular}

**. Correlation is significant at the 0.01 level (2-tailed).

*. Correlation is significant at the 0.05 level (2-tailed).

\section{Correlation between alpha-enolase and Ki67,} p53 in pancreatic cancer tissues.

It has been reported that alpha-enolase is involved in cancer proliferation and progression [5]. And, two conventional markers including Ki67 and p53 were also widely used to predict the prognosis of cancer patients. Then, we evaluated their expression in the same tissue array by IHC. The results demonstrated that high Ki67 expression was detected in 39\% (39/100), and positive p53 staining was found in $48 \%(48 / 100)$. Statistical analysis indicated that high levels of alpha-enolase were significantly associated with elevated Ki67 $(\mathrm{P}=0.02)$ and p53 $(\mathrm{P}=0.001)$ expression. Spearman correlation analysis also revealed that aberrant expression of alpha-enolase was positively associated with Ki67 expression and inversely correlated with p53 expression in PC samples (Table 3). These observations demonstrated that over-expression of alpha-enolase might play important roles in cancer progression.
Alpha-enolase expression was associated with overall survival in $\mathrm{PC}$ patients.

Kaplan-Meier analysis was used to examine if the different locations of alpha-enolase expression correlated with PC patient's survival. Our data showed that high levels of total alpha-enolase expression was significantly correlated with overall survival of PC patients $(\mathrm{p}<0.001)$. On the contrary, the expression of membranous alpha-enolase was significantly associated with better survival in pancreatic cancer patients. There was no significant correlation between high levels of ki67 or p53 and poor survival (Figure 3). Next, we performed the multivariate survival analysis by using Cox multivariate regression model. The results revealed that total alpha-enolase level $(\mathrm{HR}=2.469 ; 95 \% \mathrm{CI}$ : 1.348-4.522; $\mathrm{P}=0.003$ ) was an independent prognostic factor for pancreatic cancer (Table 4 ).

Table 4. Multivariate analysis of Cox Proportional Hazards Model for pancreatic cancer

\begin{tabular}{lllllllll}
\hline Characteristics & B & SE & Wald & df & Sig. & \multicolumn{2}{c}{$\operatorname{Exp(B)} \begin{array}{l}\text { 95.0\% CI for } \\
\text { Exp(B) }\end{array}$} \\
\cline { 6 - 9 } & & & & & & & & \multicolumn{3}{c}{ Lower } & Upper \\
\hline alpha-enolase & .904 & .309 & 8.575 & 1 & $.003^{*}$ & 2.469 & 1.348 & 4.522 \\
Stage & .543 & .513 & 1.118 & 1 & .290 & 1.721 & .629 & 4.707 \\
Lymph node & .159 & .450 & .124 & 1 & .724 & 1.172 & .485 & 2.829 \\
involvement & & & & & & & & \\
Depth of & -.456 & .367 & 1.550 & 1 & .213 & .634 & .309 & 1.300 \\
invasion & & & & & & & & \\
Grade & .304 & .398 & .584 & 1 & .445 & 1.356 & .621 & 2.960 \\
KI67 & -.187 & .276 & .457 & 1 & .499 & .830 & .483 & 1.426 \\
P53 & -.114 & .272 & .177 & 1 & .674 & .892 & .523 & 1.520 \\
\hline
\end{tabular}

\section{Discussion}

Pancreatic cancer is one of the most lethal human cancers with poor prognosis. Most pancreatic cancer patients can not be early diagnosed and lack of effective treatment [7]. Although targeted therapy has shown effectiveness against most cancers, the number of targeted drug for pancreatic cancer is really limited. Therefore, it is necessary to identify novel drug target for pancreatic cancer to achieve the best clinical outcomes.

Alpha-enolase is a glycolytic enzymes responsible for converting glucose into pyruvate. It is also involved in various pathophysiological processes such as cell growth control and immune response [8]. Overexpression of alpha-enolase in monocyte could enhance plasmin activity and transmigration into the acute lung injury tissues [9]. During hypoxia, alpha-enolase was up-regulated by HIF-1a in retinal pigment epithelial cells, which might contribute to choroidal neovascularization [10]. 
A

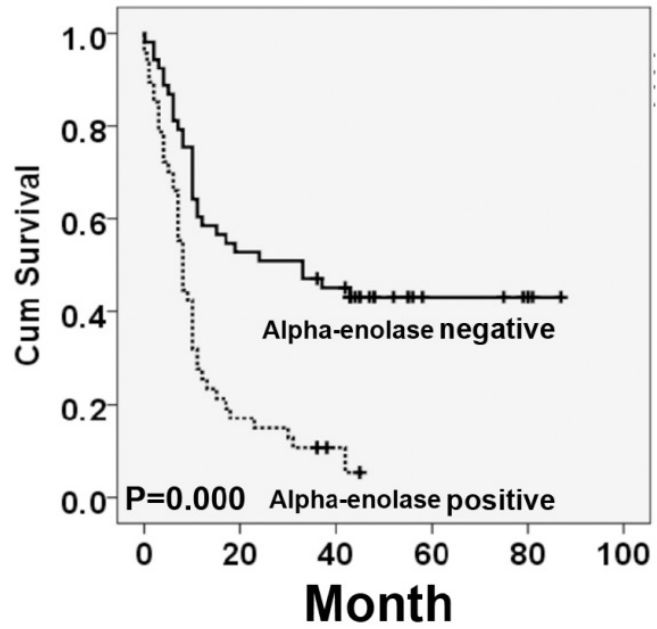

C

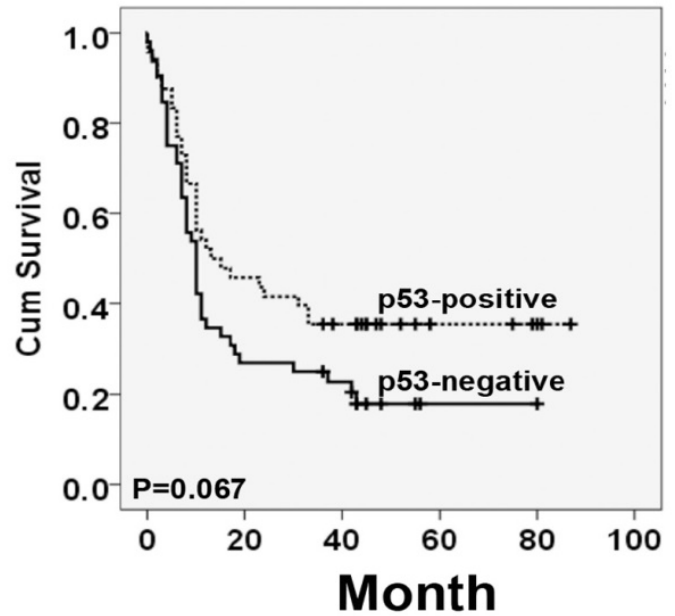

B

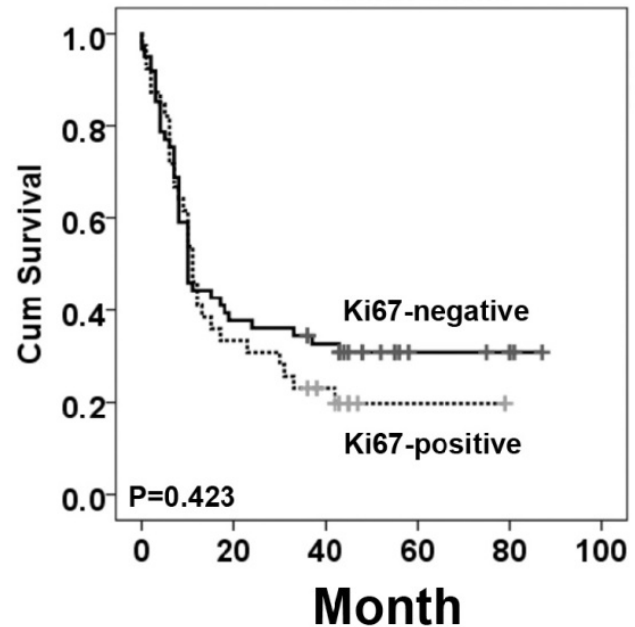

D

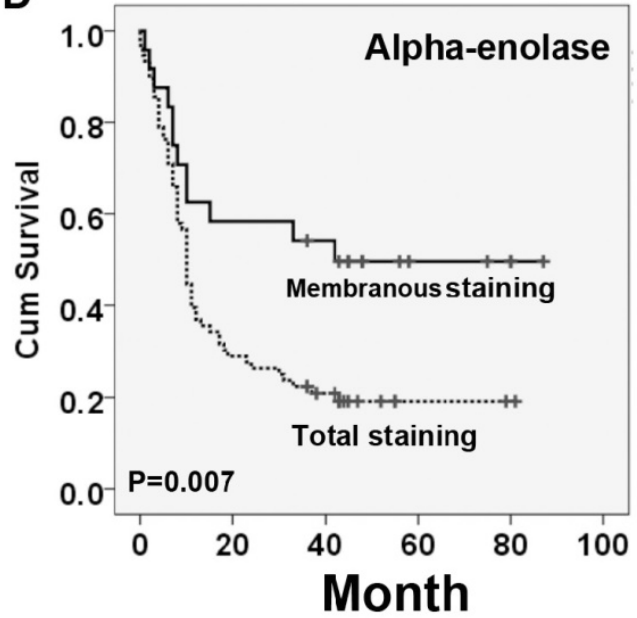

Figure 3. Survival curves for pancreatic cancer using the Kaplan-Meier method and the log-rank test. A. Overall survival curves for patients with negative Alpha-enolase expression (real line) and patients with positive Alpha-enolase (dotted line). B. Overall survival curves for patients with negative ki67 expression (real line) and patients with positive ki67 expression (dotted line). C. Overall survival curves for patients with negative p53 expression (real line) and patients with positive p53 expression (dotted line). D. Overall survival curves for patients with Alpha-enolase membranous expression (real line) and patients with Alpha-enolase total expression (dotted line).

Abnormal glycometabolism is the fundamental property of cancer cells [11]. Its dysregulation has been reported in several types of cancer, and was closely associated with cancer progression. Over-expression of alpha-enolase was found to play key roles in cancer cell proliferation and metastasis by activating FAK/PI3K/AKT pathway in non-small cell lung cancer (NSCLC) [5]. In glioma, up-regulation of alpha-enolase was responsible for cell growth, migration and invasion [3]. On the contrary, down-regulation of alpha-enolase was associated with poor overall survival in clear cell renal cell carcinoma [12]. In pancreatic cancer, alpha-enolase was found to be highly expressed in the cancer cell membrane, and alpha-enolase targeting mono-clonal antibody could significantly inhibit lung metastases in an experimental animal model [6]. Despite the potential implication of alpha-enolase for the cancer progression, no previous studies examined its level and clinical significance in pancreatic cancer tissues.

In this study, we firstly found that mRNA levels of alpha-enolase were significantly higher than normal tissue in two independent publicly available dataset in the Oncomine. Then, IHC assay revealed that alpha-enolase was highly expressed in pancreatic cancer tissues with a positive rate of $47 \%(47 / 100)$. Further statistical analysis indicated that high levels of alpha-enolase was significantly associated with Lymph node involvement and Tumor size. Previous studies proved that alpha-enolase has diverse functions depending on its localization. In this study, we found that alpha-enolase membrane positive expression rate was $29.8 \%(14 / 47)$ among the positive samples. And over-expression of membrane 
alpha-enolase was significantly associated with Lymph node involvement. It is possible that alpha-enolase, as a plasminogen receptor, could promote the plasminogen activation to facilitate cancer invasion [6]. p53 is a critical tumor suppressor protein, and its inactivation occur in most human cancers [13]. Moreover, Ki67 is a popupalr indicator for clinical pathology to estimate the tumor growth [14]. Therefore, we evaluated the expression of Ki67 and p53 in the same tissue array by IHC. The results showed that staining of Ki67 and p53 was mainly nucleus-positive, and Ki67 expression was detected in $39 \%$ (39/100) PC samples, and positive stain of p53 was found in $48 \%(48 / 100)$ specimens. Correlation analysis revealed that aberrant expression of alpha-enolase was positively associated with Ki67 and inversely correlated with p53 in pancreatic cancer tissues. These results indicated that alpha-enolase might modify cancer cell metabolism or degrade cell extracellular matrix to promote cancer progression. Then, we further proved the association between alpha-enolase abnormal expression and prognosis. We found that high alpha-enolase expression was significantly correlated with overall survival of PC patients. By contraries, membrane expression of alpha-enolase in tumor cells was significantly associated with better survival in patients with pancreatic cancer. Cox multivariate regression model demonstrated that alpha-enolase level $(\mathrm{HR}=2.469$; 95\% CI: 1.348-4.522; $\mathrm{P}=0.003$ ) was an independent predictive factor of poorer prognosis for pancreatic cancer.

In conclusion, this study for the first time demonstrated that alpha-enolase level was significantly elevated in pancreatic cancer tissues, which was closely associated with an unfavorable prognosis, and it might be a candidate target for targeted cancer treatments. Certainly, further studies should be conducted to clarify the molecular mechanism of alpha-enolase in the pancreatic cancer progression.

\section{Materials and Methods}

\section{Analysis of Oncomine Data}

To determine the expression pattern of alpha-enolase in pancreatic cancer, the datasets in Oncomine database (https://www.oncomine.org) were used. Briefly, alpha-enolase gene was queried in the database and the results were filtered by selecting pancreatic cancer and Cancer vs. Normal Analysis. The data were displayed by using Box chart. P-values for each group were calculated using student $t$-test. Details of standardized normalization techniques and statistical calculations are provided on the Oncomine.

\section{Tissue microarray and immunohistochemistry}

The commercial tissue microarrays were constructed by Shanghai Biochip Co. Ltd., as described previously [15]. Briefly, the tissue microarrays including 100 pancreatic cancer patients and 80 adjacent normal tissues were prepared from archival formalin-fixed, paraffin embedded tissue blocks. A representative tumor area was carefully selected from a H\&E-stain section. For all the specimens, clinicopathological information (age, gender, and pathology, differentiation, and TNM stage) and Follow-up information were available. Standard Avidin-biotin complex peroxidase immunohistochemical staining was performed. Briefly, after deparaffinizationin xylene and graded alcohols, heated antigen retrieval was done in citrate buffer $(10 \mathrm{mmol} / \mathrm{L} \mathrm{pH} 6.0)$ by water-bath kettle heating for $30 \mathrm{~min}$. Endogenous peroxidase was blocked in $0.3 \%$ hydrogen peroxide for $10 \mathrm{~min}$. Nonspecific binding was blocked by incubation in $10 \%$ normal animal serum for $10 \mathrm{~min}$. Sections were incubated at $4^{\circ} \mathrm{C}$ for $24 \mathrm{~h}$ with primary antibodies including polyclonal antibody against anti-alpha-enolase (ab85086, Abcam), Anti-p53 antibody (ab28, Abcam) and anti-Ki67 (ab833, Abcam). Next, biotinylated secondary antibodies and horseradish peroxidase labeled avidin were incubated with samples. Color was developed using the DAB method.

\section{Immunostaining analysis}

The tissue cores on slides were independently evaluated by 2 two pathologists who were blinded to patients' clinical data. They adopted semi-quantitative scoring system in considering the staining intensity and area extent, which has been widely accepted and used in previous studies [16]. The levels of alpha-enolase, P53 and Ki67 were scored by staining intensity and the percentage of immunoreactive cancer cells. Total staining intensity was arbitrarily scored on a scale of four grades: 0 (no staining of cancer cells), 1 (weak staining), 2 (moderate staining), and 3 (strong staining), and the percentage of positive cells was scored as follows: 0 $(0 \%), 1(1 \%$ to $50 \%), 2(51 \%$ to $80 \%)$, and $3(>80 \%)$. The staining positivity was determined using the following formula: overall score=positive percentage score $\mathrm{x}$ intensity score. For total alpha-enolase expression, a score of 0 to $\leq 3$ was defined as " 0 , Negative", and $>3$ as "1, Positive". For membrane staining score, $\leq 10 \%$ membranous staining of cancer cells was scored as "0, Negative", and >10\% membranous staining of cancer cells was scored as " 1 , positive". For Ki67 or p53, a score of 0 to $\leq 1$ was 
defined as "0, Negative", and $>1$ as "1, Positive". The interobserver variation was below $5 \%$.

\section{Statistical Analysis}

The SPSS 15 software package (SPSS, Inc., Chicago, IL) was used for statistical analysis. The association between the immunoreactive markers and clinicopathologic features was analyzed using $x^{2}$-test or two-sided t-test as appropriate. The log-rank test was performed to examine the association of alpha-enolase with overall survival.

Cox regression model was used to analyze the significance of various variables for survival. Spearman's rank correlation coefficient and Fisher's exact test were used to explore the association among alpha-enolase, p53 and Ki67 expression. All comparisons were two-tailed, and $p<0.05$ was considered significant.

\section{Acknowledgement}

This work was supported by National Natural Science Foundation of China (No. 81101625), National High-tech R\&D Program of China for Young Scholars (No.2014AA020537), Beijing Talents Fund (No. 2015000021223ZK23), Beijing Gao Chuang Ji Hua (No.G02060050), Beijing Nova Program (No.Z1511000003150121).

\section{Competing Interests}

The authors have declared that no competing interest exists.

\section{References}

1. Pandol S, Gukovskaya A, Edderkoui M, Dawson D, Eibl G, Lugea A. Epidemiology, risk factors, and the promotion of pancreatic cancer: Role of the stellate cell. Journal of Gastroenterology and Hepatology. 2012; 27: 127-34.

2. Romond EH, Perez EA, Bryant J, Suman VJ, Geyer CE, Jr., Davidson NE, et al. Trastuzumab plus adjuvant chemotherapy for operable HER2-positive breast cancer. N Engl J Med. 2005; 353: 1673-84.

3. Song Y, Luo Q, Long $\mathrm{H}, \mathrm{Hu} \mathrm{Z}$, Que T, Zhang Xa, et al. Alpha-enolase as a potential cancer prognostic marker promotes cell growth, migration, and invasion in glioma. Molecular Cancer. 2014; 13: 65-

4. Zhu X, Miao X, Wu Y, Li C, Guo Y, Liu Y, et al. ENO1 promotes tumor proliferation and cell adhesion mediated drug resistance (CAM-DR) in Non-Hodgkin's Lymphomas. Experimental cell research. 2015; 335: 216-23.

5. Fu Q-F, Liu Y, Fan Y, Hua S-N, Qu H-Y, Dong S-W, et al. Alpha-enolase promotes cell glycolysis, growth, migration, and invasion in non-small cell lung cancer through FAK-mediated PI3K/AKT pathway. Journal of Hematology \& Oncology. 2015; 8: 22.

6. Principe M, Ceruti P, Shih NY, Chattaragada MS, Rolla S, Conti L, et al. Targeting of surface alpha-enolase inhibits the invasiveness of pancreatic cancer cells. Oncotarget. 2015; 6: 11098-113.

7. Eguia V, Gonda TA, Saif MW. Early detection of pancreatic cancer. JOP. 2012; 13: $131-4$

8. Aaronson RM, Graven KK, Tucci M, McDonald RJ, Farber HW. Non-neuronal Enolase Is an Endothelial Hypoxic Stress Protein. Journal of Biological Chemistry. 1995; 270: 27752-7.

9. Wygrecka M, Marsh LM, Morty RE, Henneke I, Guenther A, Lohmeyer J, et al. Enolase-1 promotes plasminogen-mediated recruitment of monocytes to the acutely inflamed lung. Blood. 2009; 113: 5588-98.

10. Zheng F, Jang W-C, Fung FKC, Lo ACY, Wong IYH. Up-Regulation of ENO1 by HIF-1a in Retinal Pigment Epithelial Cells after Hypoxic Challenge Is Not Involved in the Regulation of VEGF Secretion. PLoS ONE. 2016; 11: e0147961.

11. Moreno-Sanchez R, Rodriguez-Enriquez S, Marin-Hernandez A, Saavedra E. Energy metabolism in tumor cells. FEBS J. 2007; 274: 1393-418.
12. White-Al Habeeb NM, Di Meo A, Scorilas A, Rotondo F, Masui O, Seivwright A, et al. Alpha-enolase is a potential prognostic marker in clear cell renal cell carcinoma. Clinical \& Experimental Metastasis. 2015; 32: 531-41.

13. Olivier M, Hollstein M, Hainaut P. TP53 Mutations in Human Cancers: Origins, Consequences, and Clinical Use. Cold Spring Harbor Perspectives in Biology. 2010; 2: a001008.

14. Dowsett M, Nielsen TO, A'Hern R, Bartlett J, Coombes RC, Cuzick J, et al Assessment of Ki67 in Breast Cancer: Recommendations from the International Ki67 in Breast Cancer Working Group. JNCI Journal of the National Cancer Institute. 2011; 103: 1656-64.

15. Zhang Z, Wang J, Ji D, Wang C, Liu R, Wu Z, et al. Functional Genetic Approach Identifies MET, HER3, IGF1R, INSR Pathways as Determinants of Lapatinib Unresponsiveness in HER2-Positive Gastric Cancer. Clinical Cancer Research. 2014; 20: 4559-73.

16. Sun L, Hu H, Peng L, Zhou Z, Zhao X, Pan J, et al. P-Cadherin Promotes Liver Metastasis and Is Associated with Poor Prognosis in Colon Cancer. The American journal of pathology. 2011; 179: 380-90. 